\title{
A Stereotaxic Headholder for Small Birds
}

\author{
HANS-JOACHIM BISCHOF \\ University of Bielefeld, Department of Biology, Ethologie \\ P.O. Box 8640, 4800 Bielefeld 1, F.R.G.
}

Received 18 July 1981

\begin{abstract}
BISCHOF, H.-J. A stereotaxic headholder for small birds. BRAIN RES. BULL. 7(4) 435-436, 1981,--A stereotaxic headholder especially designed for small birds is described. The major advantage of the holder is its easy handling when mounting the bird to the apparatus, and better access to the visual field of the bird when compared with other frequently used headholders.
\end{abstract}

Stereotaxic headholder Birds Zebra finches

MOST groups working on avian brains use head holders adapted to their needs from conventional available ones such as the Kopf and Wells stereotaxic devices. One example is the so-called Revzin-adaptor described by Karten and Hodos [1]. An advantage of their modification is the more accurate placement of the head in an exactly defined angle to the vertical, but this hinders free access to the lateral parts of the brain and restricts heavily the visual field of the bird. Another device is described by Vowles et al. [4] which is only slightly modified from the Kopf and Wells stereotaxic apparatus. It also accurately fixates the head of the bird, but does not seem to be appropriate for small birds, as the second fixating point, the lachrymal duct, is too tiny and fragile in these animals.

The most important inconvenience of these headholders is the difficulty in accurately centering the head in the apparatus, as one has to adjust two different earbars. The procudure which has to be accomplished to fix the head in the correct angle by adjusting the mouth bar is also difficult and time consuming. The device described below avoids these two disadvantages. It was originally developed for experiments with zebra finches (Taeniopygia guttata castanotis Gould).

The head holder is made from aluminum, which is stable enough for the small animals. It is mounted on an aluminum plate of $20 \times 8 \mathrm{~cm}$. This plate can in turn be affixed to a solid steel plate which has a rail for the stereotaxic microdrive (Fig. 1).

The construction of ths headholder is demonstrated in Fig. 2. The rotatable two earbars (E) are inserted into the main block $(M)$ of the device. They can be moved like a pair of tongs by turning a spindle drive (S) located under the main block. The up-down movement of the spindle block is transferred to the earbars by two levers which connect block and earbars by articulated joints.

The tip of the bird's beak was chosen as the second fixating point. It was fixed by the beak holder (B) which is also movable. The movable part is inserted into a hole in the main block which is drilled in a direction so that its axis meets the imaginary line between the two earbars and builds an angle of 45 degrees to the vertical. The beak holder can be moved in the directions determined by the drill hole and can be fixed by a screw (F). At the tip the beak holder has a cone shaped hole for the insertion of the beak tip.

The following steps should be followed to get the bird's head into the stereotaxic apparatus: First the body is placed into the tube $(T)$; then the head is placed between the two earbars. The earbars are clamped to the ears by rotation of the spindle drive. When the earbars are fixed carefully, the beak tip has to be inserted into the cone hole of the beak holder by pushing the bar towards the beak as far as possible. One has to be very careful to meet the ear holes with the ear bars. Incorrect mounting has occurred if the beak does not fit easily into the cone hole of the holder.

The beak tip was chosen as the second fixation point as it is easier to attach the beak holder to the bird than a mouth bar. In our strain of zebra finches the accuracy provided by the described device proved to be good. We have tested the headholder in about 200 stereotaxic experiment, together with our atlas of the zebra finch brain (Bischof and Nixdorf, unpublished observations), and we hit the desired structures in about $80 \%$ of all trials, which seems to be very good when one takes into consideration the uncertainties involved e.g. the estimation of the zero point for the stereotaxic coordinates, or the variability of the brain size). For birds with larger variability of the beak size or for adaptation of the device for small mammals, the beak holder can be easily replaced by a mouth bar.

The dimensions of the headholder might have to be changed, however, if the birds are very different in size compared with zebra finches. One has to be aware that the earbars do not move on a straight line but on an orbit; with large variations in the ear distance, the elevation of the earbars and therefore the angle between the beak tip-earbar axis and the vertical changes slightly.

Other changes of the device may be possible due to the needs of the experiments. For histological work the angle of 45 degrees between the ear-beak tip axis and the vertical might be best for easier comparison and sections with the results of other birds, e.g. , the pigeon[1], the chick [3], or the 


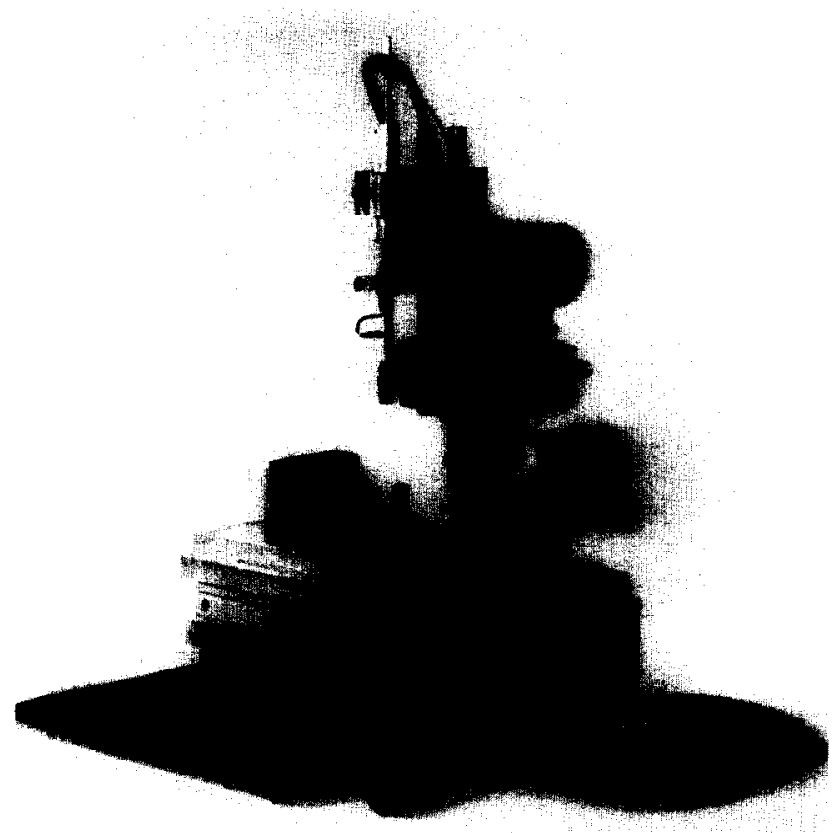

FIG. 1. Stereotaxic device with headholder, which is mounted on an additional cross drive, three-dimensional microdrive, electrode holder and probe.
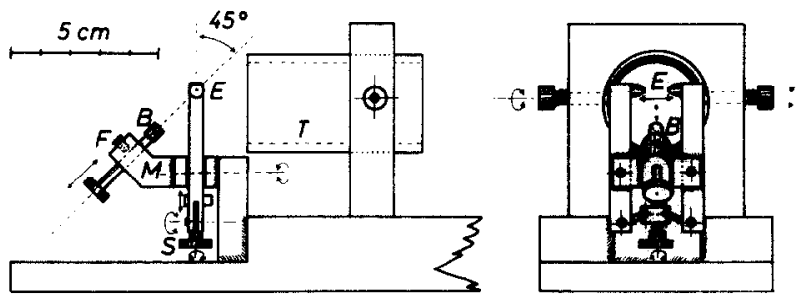

FIG. 2. The headholder in lateral and frontal view. $M=$ main block, $\mathrm{B}=$ beak holder, $\mathrm{F}=$ fixating screw, $\mathrm{E}=$ earbars, $\mathrm{S}=$ spindle drive, $\mathrm{T}=$ tube, $\odot=$ pivots.

canary [2]. For electrophysiological research it might be better to adapt the posture of the head to that normally observed in the species. This facilitates the interpretation of receptive field properties and eventually avoids influences of the vestibular system, which might react in a wrong way if the head is tilted from its normal position.

\section{REFERENCES}

1. Karten, H. J. and W. Hodos. A Stereotaxic Atlas of the Brain of the Pigeon (Columbia livia). Baltimore: Johns Hopkins Press, 1967.

2. Stokes, T. M., C. M. Leonhard and F. Nottebohm. The telencephalon, diencephalon, and mesencephalon of the canary, Serinus canaria, in stereotaxic coordinates. J. comp. Neurol. 156: $357-374,1972$.
3. van Tienhoven, A. and L. P. Juhasz. The chicken telencephalon, diencephalon, and mesencephalon in stereotaxic coordinates. $J$. comp. Neurol. 118: 185-197, 1962.

4. Vowles, D. M., L. Beazley and H. D. Harwood. A stereotaxic atlas of the barbary dove (Streptopelia risoria). In: Neural and Endocrine Aspects of Behavior in Birds, edited by P. Wright, P. G. Caryl and D. M. Vowles. Amsterdam: Elsevier, 1975. 